\title{
Un precinto de plomo aparecido en Nina Alta, Teba (Málaga)
}

\author{
A seal of lead from the deposit of Nina Alta in Teba (Malaga) \\ Pilar Delgado Blasco*
}

\begin{abstract}
RESUMEN
En el Museo Municipal de Teba (Málaga) y procedente del yacimiento arqueológico de Nina Alta (Málaga) hay expuesto un precinto de plomo con la leyenda en árabe mușālaḥa, o pacto de paz, que hace referencia a la conquista islámica del 711 . Se trata de un sello ligado a un tratado de paz y que ha permanecido inédito hasta este momento. Puede ser uno de los primeros hallazgos materiales de la conquista que podamos relacionar con Tākurunnā.
\end{abstract}

Palabras clave: Precinto de plomo, Pacto de paz, alAndalus, Conquista islámica, Nina Alta, Tākurunnā.

El museo municipal de Teba (Málaga) alberga una importante colección de piezas de distintas épocas procedentes de un yacimiento arqueológico cercano llamado Nina Alta. Entre ellas se encuentra un precinto de plomo que, por sus características, ha de ser puesto en relación con los ejemplares que han sido datados en los albores de la conquista de Hispania por los musulmanes.

Existen numerosas publicaciones sobre este hecho histórico ${ }^{1}$, pero con respecto a su materialidad las únicas huellas conocidas hasta hace escasas décadas son los precintos de plomo y las monedas. Durante mucho tiempo, solamente las fuentes escritas habían servido para explicar los acontecimientos ocurridos durante el paso de los conquistadores por la península ibérica y la Narbonense.

Al hilo de esto y como bien dice A. García Sanjuán (2013: p.152), el año 711 carece de una

\begin{abstract}
In the Municipal Museum of Teba (Málaga) and coming from the archaeological site of Nina Alta (Málaga) there is a lead seal with the legend in Arabic of mușālaha, or peace pact, which refers to the Islamic conquest of 711 . It is a seal linked to a peace treaty and has remained unpublished until now. It may be one of the first material findings of the conquest that we can relate to Tākurunnā.
\end{abstract}

Keywords: Lead Seal, Peace Pact, al- Andalus, Islamic Conquest, Nina Alta, Tākurunnā.

realidad arqueológica precisa y no sería lógico que desde la arqueología pretendiésemos documentar la conquista de Hispania. Pero esto no significa que descartemos el registro material referido a esta época: tanto sellos como monedas, aunque relativamente escasos, tienen un valor inapreciable al representar las únicas manifestaciones de las exigencias de los nuevos dominadores que consistían, sucintamente, en reservar un quinto o jums de las tierras para ponerlas bajo la administración directa de la comunidad representada legítimamente por los soberanos omeyas, según el derecho islámico, en caso de conquista por las armas (MARTíNEZ NÚÑEZ, 2011: 25). Mientras, en caso de capitulación lo que se justificaba era el requerimiento del pago de impuestos.

Parece pues que la percepción sobre el hecho de la conquista está "disponible" en unas cuantas monedas de cuño coetáneo al momento de la invasión y en los precintos

\footnotetext{
*Arqueóloga. Museo de Ronda. Empresa municipal de Turismo de Ronda (Málaga), Museo de Ronda-Palacio de Mondragón. Pl. de Mondragón s/n 29400 Ronda (Málaga), mpilardelgado@gmail.com, pilardelgadoblasco@uma.es, Orcid: 0000-0002-9790-0691

1. Sin afán de exhaustividad, entre las más reseñables se pueden citar las siguientes: COLLINS, (1991); CHALMETA GENDRÓN, (1994); MANZANO MORENO (2000); GUICHARD (2002); GARCÍA SANJUÁN (2004 y 2013); KENNEDY (2007); DOMENÉ SÁNCHEZ (2011); ORTEGA ORTEGA (2018).
} 
plúmbeos relacionados con el reparto del botín y el pago de impuestos. Además, constituyen el primer documento histórico referente al uso de la lengua árabe en la península ibérica (GUTIÉRREZ LLORET, 2011: 191).

A diferencia de otras fuentes escritas, estos testimonios epigráficos no se concibieron para reflejar el acontecimiento histórico de la conquista ni para influir en las teorías sobre este momento, no tenían esa funcionalidad, sin embargo tienen una relevancia histórica extraordinaria (MARTíNEZ NÚÑEZ, 1997: 128).

Pero, con respecto a las fuentes materiales, no debemos perder de vista la importancia de los estudios realizados sobre los restos aparecidos en la maqbara de Pamplona, en la Marca Superior, relacionados de forma clara con la invasión². La presencia de anillos signatarios con inscripciones en cúfico nos muestra la evidencia de los primeros contactos entre la población del norte peninsulary las gentes que cruzaron el Estrecho, vinculadas culturalmente al Islam (DE MIGUEL IBAÑEZ, 2016: 71). La incorporación de este tipo de piezas de adorno aparecidas en las sepulturas de algunos individuos de los cementerios pamplonicas con fórmulas religiosas en árabe, remite a un estatus social alto (MARTÍNEZ NÚÑEZ, 2011: 185)³), inclusive pudiera relacionarse con alguna de las consecuencias del Pacto de Pamplona (DE MIGUEL IBÁNEZ, 2016: 43).

Como vemos, la difusión de la religión y de la lengua de los conquistadores se convertirá en factor importante para esta nueva sociedad. Una y otra se van a concretar a través de diferentes expresiones; la primera con la edilicia y con los ritos religiosos y la segunda, con los soportes propicios para la escritura como los sellos y las monedas que, a su vez, poseen un doble valor, el propagandístico (CANTO, IBRAHIM, 2004: 22 y 53) y el histórico, siendo la aportación empírica más importante sobre el acontecimiento de la conquista musulmana que se ha producido desde el siglo XIX (GARCÍA SANJUÁN, 2013: 168) ${ }^{4}$.

En ambos soportes la epigrafía nos proporciona varios niveles de información; uno derivado de su aspecto formal y de los rasgos caligráficos, mientras que el otro nos lo facilita el contenido de los textos grabados. En estos casos cabe recordar que el Estado se expresa en árabe a través de ellos siendo además esta la lengua vehicular de los conquistadores que, a partir de estos momentos, se asientan en la península ibérica y, aun cuando estemos ante el punto de partida de lo que fue al-Andalus, es importante tener en cuenta que los precintos encontrados, al contrario que algunas de las primeras monedas que eran bilingües, solo aparecen escritos en árabe (MARTíNEZ NÚÑ̃EZ, 2001: 22).

Sobre las monedas, los llamados "dinares bilingües" del año 98 H/716 d. C, son los primeros documentos históricos fechados en los que se usa la lengua árabe en la península ibérica. Solo un año antes se acuñaron en el Magreb, pero presentan algunas diferencias. Estas monedas áureas son de tradición bizantina y en ellas se sustituyeron las leyendas de carácter político por los mensajes de contenido religioso referidos principalmente a la unicidad de Dios (ARIZA ARMADA, 2017: 95).

Obviamente, y al hablar de estos soportes, debemos resaltar que, con respecto al topónimo al-Andalus, aparece antes en los

\footnotetext{
2. La capitulación de este territorio se produjo siendo gobernador 'Abd al- 'Azīz Ibn Mūsà. Así, este sitio de vascones queda vinculado a la autoridad islámica desde el año 714 hasta el 718. En las intervenciones arqueológicas realizadas en Argaray primero, y más recientemente en la Casa del Condestable, los arqueólogos hallaron, en algunas de las sepulturas de ritual cristiano, anillos con inscripciones en árabe. Este hecho hay que ponerlo en relación con la presencia de contingentes árabo-beréberes. Las pruebas de datación indicaron una fecha posterior al 714 y se fecharon entre el 715 y el 770 (DE MIGUEL 2016: 134 y 634), y los análisis paleopatológicos remiten a grupos alóctonos procedentes del Norte de África.

3. En algunos de los anillos de la Casa del Condestable aparece una expresión coránica (Q. IX, 129/ XXXIX, 38) hasbī Allāh (Dios me basta).

4. Véase también sobre estas monedas: BATES (1992); CANTO, IBRAHIM (2004); CANTO GARCÍA (2011); IBRAHIM (2011).
} 
precintos que en las monedas (IBRAHIM, 2011: 149; IBRAHIM, 2016: 16)5 $)^{5}$ ya que las primeras acuñaciones llevan la ceca de SPaNia hasta que se produce el cambio de capitalidad que hace al-Hurr desde Sevilla a Córdoba. El cambio no es baladí y estará entroncado con el control político y administrativo que confirma a la autoridad que pone en circulación la moneda, en este caso la islámica.

Y algo más que también cabe destacar es que la introducción de las leyendas en árabe puede ser un atisbo del proceso de arabización del Magreb y, tal vez, de la consolidación del árabe como la lengua del Islam en general (ARIZA ARMADA, 2016: 142).

\section{LA IMPORTANCIA DE LOS PRECINTOS DE PLOMO}

Como hemos dicho, hay dos elementos materiales ligados irremediablemente a la conquista: las monedas y los precintos. Desde el punto de vista formal son unas piezas de plomo, casi siempre de forma irregular, con dimensiones distintas, cuyo único vínculo formal común es que en ellas siempre aparece una leyenda escrita en caracteres cúficos. Estas piezas se ataban a sacos mediante un alambre o un cordel y así eran sellados ${ }^{6}$.

Con respecto a su materialidad, mientras que las monedas tienen una doble función, la del objeto de uso como medida de valor y medio de pago, y la de elemento propagandístico, ya que simboliza el poder del Estado en todo su sentido con un uso perdurable, los precintos de pacto están relacionados en su mayor parte con la imposición de una nueva fiscalidad?. En este sentido, expresan la voluntad del nuevo Estado y representan la materialidad de un acto ejecutado en un momento puntual.

Los términos que podemos observar en los precintos indican cómo se apropiaron de ese territorio los conquistadores. Así por ejemplo, maqsūm, "lo que ha sido repartido", o magnūm țayyib, "botín lícito", son expresiones referidas a un territorio conquistado por las armas, o 'unwatan, lo que se relaciona significativamente con el reparto del botín, que significa que todos los bienes eran confiscados a sus propietarios y perdían todo derecho sobre ellos (IBRAHIM, 2011; LORENZO JIMÉNEZ, 2011: 28).

Por el contrario, las palabras șulh/mușālaha aluden a un tratado de paz o capitulación, hecho, en ocasiones, en nombre del califa omeya de Damasco al-Walīd I (705-715 d. C) (IBRAHIM, 2011: 151, IBRAHIM, 2016: 22, MARTÍNEZ NÚÑEZ, 2011: 21 y 25) y, por lo tanto, al acatamiento de algún tipo de condiciones implícitas al propio pacto que fueron de obligado cumplimiento para los habitantes de los lugares conquistados, como es el pago de la yizya (impuesto de capitación), que se convirtieron en cláusulas ineludibles para las poblaciones de asentamientos sometidos mediante pacto, a cambio de poder conservar vida, bienes y creencias (GUTIÉRREZ LLORET, 2011: 197) ${ }^{8}$.

Así parece que fue lo que sucedió en la mayor parte de Hispania, que se supeditó

\footnotetext{
5. Alude al precinto en el que se documenta la más arcaica mención de al-Andalus, resellado en época de al-Hurr.

6. Los precintos de la conquista, sus características, tipos y significado histórico han sido dados a conocer y estudiados en sucesivas publicaciones, en primer lugar por T. Ibrahim y por este y otros autores, como GASPARIÑO, IBRAHIM (2015 y 2019), en las páginas de la revista Manquso. Los procedentes de la Narbonense por SÉNAC (2012); SÉNAC et alii (2014) y recientemente Ibrahim y Sénac han colaborado en varias publicaciones, como SÉNAC, IBRAHIM (2017a), SÉNAC, IBRAHIM (2017), Los precintos de la conquista, 2017b; CHALMETA GENDRÓN (2015). Este último en su artículo sobre "Los primeros 46 años de economía andalusí" nos dice: Obsérvese que esta capitulación/șulh (a) impone una tributación "híbrida", puesto que engloba una entrega valorada en términos de moneda más otra en productos de la tierra; administrativamente lo exigido es el pago de un tributo personal/ŷizya más una contribución territorial/ jarāŷy: el Pacto de Teodomiro está pensado para una población rural. (b) La cuantía final del pago viene condicionada por el número de contribuyentes. (c) Es un prorrateo donde los terratenientes son globalmente clasificados como pobres, tributando a lo más bajo, y los siervos/colonos (asimilados a pequeños propietarios agrícolas) pagan la mitad. (d) Aunque no señala quién es el recaudador, es evidente que se trata del propio Tudmir. (CHALMETA GENDRÓN, 2015: 64).

7. Musā b. Nușayr fue el artífice de la instauración de un sistema fiscal sólido y eficaz (CHALMETA GENDRÓN 2015: 45).

8. De hecho, algunas iglesias se mantienen bajo el amparo de los pactos, como así se ha podido comprobar en el pacto de Tudmīr.
} 
conforme a acuerdos de capitulación mediante pactos de dimma ${ }^{9}$, cuyos firmantes fueron los terratenientes locales (CHALMETA GENDRÓN, 2015: 45). (Fig. 1)

Este tipo de tratados de sulh o musālaha tienen implicaciones tanto jurídicas como fiscales. Los pobladores indígenas adquieren el estatuto de protegido o dimmi por el que mantenían sus leyes y creencias, mientras con respecto al tema fiscal se establecen una serie de tributos como se recoge en el conocido pacto Tudmîr, del que no es descartable que aparezcan sellos de sulh a nombre de Tudmir, lo que permitirá tener un conocimiento de la fiscalidad mucho más completo (GUTIÉRREZ LLORET, 2014: 275).

Hay dos zonas a las que se pueden circunscribir los precintos conocidos hasta el momento. Una es la región gala de la
Narbonense, concretamente Ruscino, cerca de la actual Perpiñán, en la que hace unos años salió a la luz una importante colección de sellos de plomo, y la otra es el sur de la península Ibérica. Casi todos ellos han sido datados en la primera mitad del siglo VIII, y gran parte recopilados y publicados por Ph. Sénac y T. Ibrahim (SÉNAC, IBRAHIM, 2017a y b). Entre los encontrados en esta zona meridional francesa y los peninsulares suman más de un centenar ${ }^{10}$.

En algunos sellos aparecen los nombres de emires de al-Andalus, con términos asociados a ellos, por ejemplo, al-Hurr y la palabra qism (lote o reparto), al-Samh y șulh (tratado), 'Anbasa, en cuya leyenda se consigna la expresión "esto es lo que ordenó" o 'Abd al-Rahmān b. Mu'āwiya cuyo texto hace referencia al ŷayš (ejército) del emir o a un habus sobre una cota de malla propiedad del emir y el mismo sello lleva la impronta de este tipo de armadura

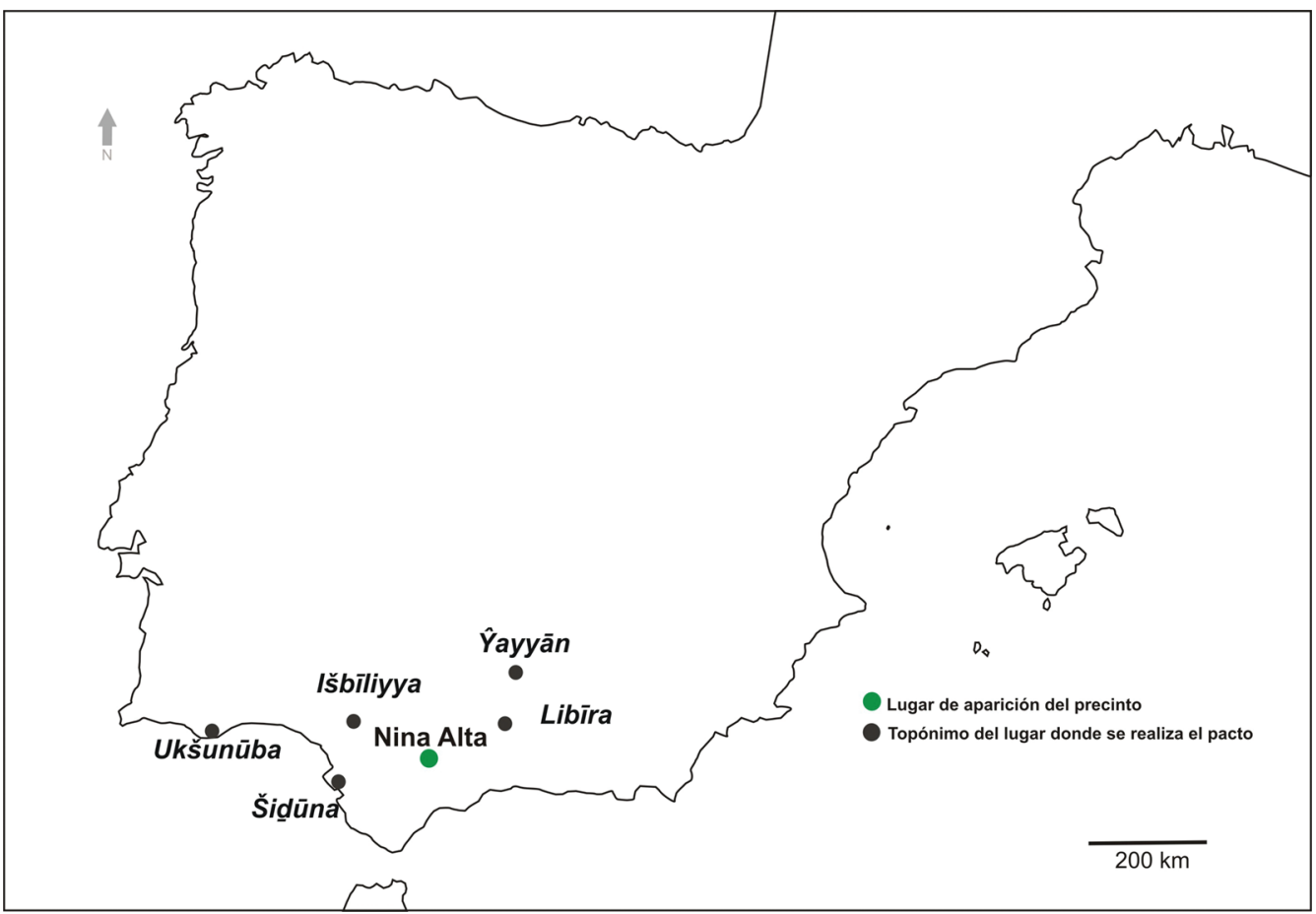

Fig. 1. Mapa de precintos de pacto de paz o șulh/ mușālaḥa. Elaboración propia.

9. "Ese pacto entre los musulmanes y los no musulmanes que habían recibido una revelación (las "gentes del Libro" o ahl al-kitāb) aseguraba la libertad de creencia, pero imponía restricciones de tipo económico, social y político a los no musulmanes: no implicaba persecución, pero sí discriminación” (FIERRO, 2018)

10. A diferencia de los peninsulares, los plomos de la Narbonense se descubrieron hace más de una década durante una prospección sistemática llevada a cabo con detector de metales y autorizada por el Servicio Regional de Arqueología de esta región francesa (RÉBE, RAYNAUD, SÉNAC 2015: 277-288; SÉNAC, IBRAHIM 2017a: 22). 
(IBRAHIM, 1995: 146; IBRAHIM, 2011: 159, f. 18, SÉNAC, IBRAHIM, 2017b: 647 y 656; LABARTA GÓMEZ, 2016: 276$)^{11}$.

En estas piezas es importante resaltar la presencia de términos derivados de la raíz slh, tanto en sulh como musālaha, que amparan el cuerpo de rendición establecido por los conquistadores sobre los vencidos.

Algunos de estos sellos van asociados al nombre del lugar que suscribió el pacto y a sus moradores, que tenían la obligación de cumplirlo, ahl Išbïliyya (gente de Sevilla) o jātim ahl Bāŷa (sello de la gente de Beja), además de otros términos como daqĩa (harina), que alude a lo que portaba la bolsa precintada, daqīa Bāŷa, harina de Beja (SÉNAC, IBRAHIM, 2017: 93).

Hay otro lote de plomos que hacen referencia al reparto del botín o qism, mencionando la división o la distribución de lo obtenido. También qusima (se ha repartido), maqsūm tayyib o reparto lícito (SÉNAC, IBRAHIM, 2017: 100 y 129), o la validez del citado reparto, como podemos ver a través de los términos, ŷawaz y aŷaza (lícito).

Los que se refieren a la conquista por las armas suelen llevar aparejado el término magnūm tayyib (botín lícito), todos ellos con el topónimo de Arbūna, Narbona, (SÉNAC, IBRAHIM, 2017a: 105), lo que resulta curioso, puesto que en los sellos peninsulares descubiertos no hay ninguno en el que se haya podido leer el término magnūm (botín), aunque esto no significa que no hubiese lugares que se conquistaran por la fuerza.

Muy al contrario, pues en los precintos sureños hay algunos concernientes al reparto de los ingresos procedentes del quinto del botín con el vocablo fay' (CHALMETA GENDRÓN, 2013: 82) y como ejemplos tenemos: fay' Allāh Rayyo (GASPARIÑO, IBRAHIM, 2015: 7) o fay' Allāh al-Andalus (SÉNAC, IBRAHIM, 2017a: 114).
Como hemos visto, muchos de ellos, además, aluden al lugar donde se ha realizado la capitulación o donde se ha hecho el pago del impuesto o del reparto del botín. Podemos ver el nombre de lugares como al-Andalus, Rayyo, Šiduna, Išbïliyya, Ilbirra o Ŷayyān, entre otras poblaciones.

Todo lo que venimos exponiendo hasta aquí se justifica porque el sello de plomo del Museo de Teba es la única pieza de estas características aparecida, por el momento, en esta comarca que está relacionada con la conquista, y tenemos que destacar que cumple varios de los requisitos para convertirse en unas de las claves para el conocimiento de lo que ocurrió en el año 711 y siguientes en lo que luego fue Tākurunnā. Como fuente material, nos proporciona los niveles de información a través de su caligrafía y de su lectura. Pero, a la vez, debemos tener en cuenta que el precinto, del que sí conocemos su procedencia, nos está indicando que en ese lugar posiblemente se llegó a un pacto por parte de sus habitantes tras la llegada de los omeyas. El hándicap principal es que la pieza no se halló durante una intervención arqueológica, y esta circunstancia resta mucha información sobre su contexto arqueológico, lo que sería fundamental para el análisis de este tipo de piezas, y por ello hay que ser prudente ${ }^{12}$

\section{EL PRECINTO HALLADO EN NINA ALTA (TEBA)}

El precinto objeto de este estudio fue donado en 1998 al museo de Teba por un vecino, tras un hallazgo casual ${ }^{13}$, y sabemos por la información existente en el Museo Municipal de Teba que, efectivamente, su procedencia es este yacimiento (Fig. 2).

De él hay bastantes más piezas depositadas en el museo local que parecen relevantes, tanto desde el punto de vista arqueológico

11. El relieve de la cota de malla es la única referencia material disponible sobre cómo eran estas protecciones en época andalusí. 12. En Ruscino ocurre algo similar (SÉNAC et alii, 2014: 64).

13. Ley 14/2007 de 26 de noviembre del Patrimonio Histórico de Andalucía, art. 50. 


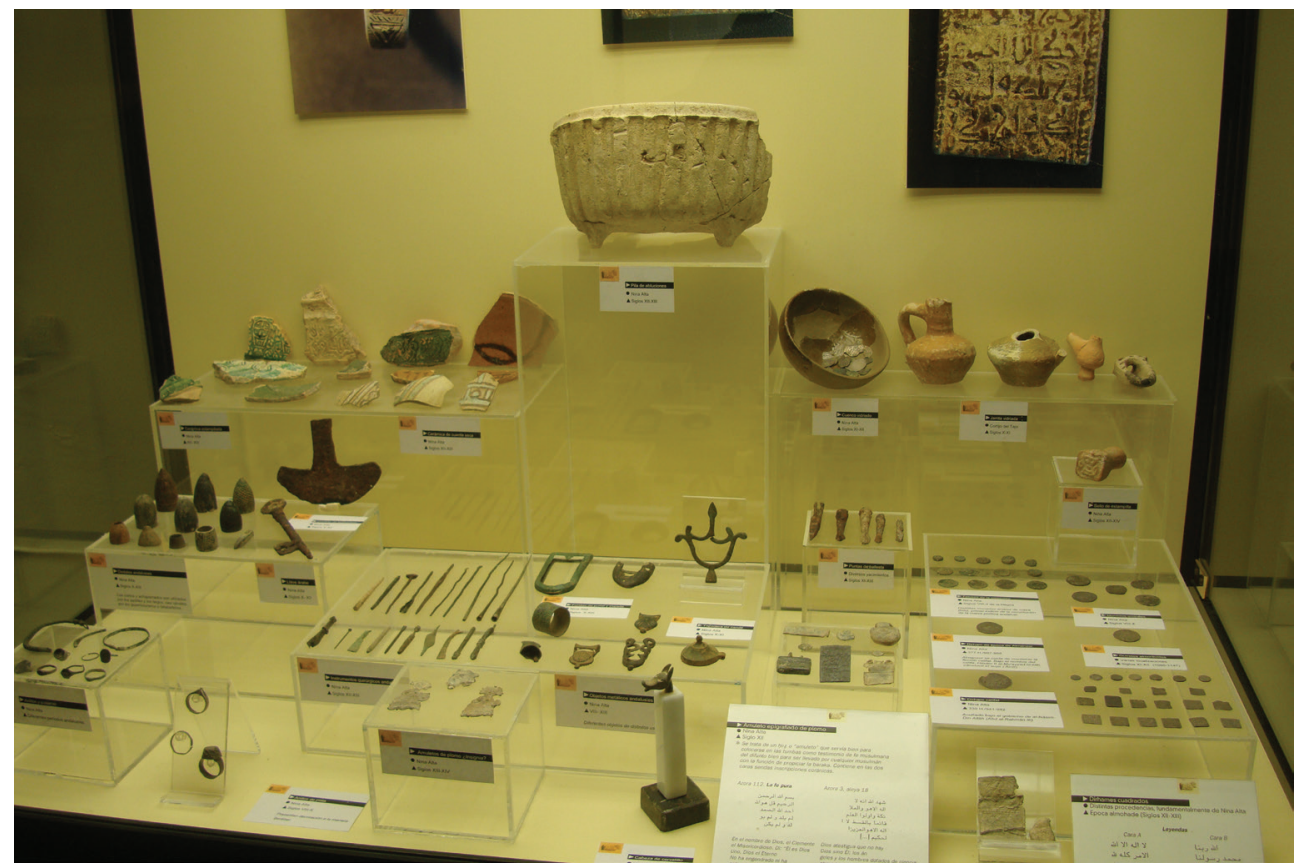

Fig. 2. Vitrina del Museo de Teba donde se encuentran piezas de época medieval procedentes de Nina Alta (https://viajeaningunlugar.wordpress.com/2014/12/08/ museo-arqueologico-de-teba-malaga/).

como epigráfico, aunque solo poseemos meras referencias sobre ellas en un par de publicaciones (MORGADO, MARTíNEZ, GARCÍA, 2001; MARTÍNEZ ENAMORADO, 2004). Pero, por la importancia que posee la pieza como posible marcador del proceso de conquista en esta zona, hay que insistir en que sería de mucho interés que se pudiese realizar un trabajo de campo y un estudio sobre este yacimiento arqueológico. Debemos decir que esta pieza ha permanecido inédita hasta el momento de redactar este trabajo ${ }^{14}$.

\section{CARACTERÍSTICAS DE LA PIEZA}

La importancia que los precintos de plomo han ido adquiriendo en la bibliografía especializada queda de manifiesto no solo por las numerosas publicaciones centradas en muchas de estas piezas, sino por la recopilación más actualizada de estos objetos, realizada, además, por las personas que específicamente se han dedicado a este tema. En la monografía sobre el particular, elaborada por Sénac e Ibrahim (2017a), se recogen 18 sellos con la misma leyenda, mușālaha (دصدالحة), que la grabada en el que aquí se estudia, aunque, a diferencia de este, en aquellos aparece el topónimo del lugar sobre el que recae el tratado de capitulación, como Ilbīra/ Libīra, Akšūniya/Ukšunūba, Šid̄ūna, Ŷayyān, o Išbīliyya (SÉNAC, IBRAHIM, 2017: 81a 87).

El precinto de Nina Alta es una placa de plomo casi circular, de la que se conserva

14. Quiero manifestar mi agradecimiento al Dr. Serafín Becerra Martín porque fue él quien nos llamó la atención sobre la existencia de este sello en el Museo Municipal de Teba, también por su inestimable ayuda y por el interés que siempre le ha prestado al conocimiento de la Historia y del Patrimonio de esta comarca malagueña.

Los datos han sido facilitados por D. José Carlos Escalante Gil, responsable del Museo Municipal de Teba, al que tengo que dar las gracias por su amabilidad y su presteza, sobre todo en los momentos en los que redacté el texto, ya que nos encontrábamos en situación de estado de alarma. También agradezco al personal del ayuntamiento de esta localidad su amabilidad, y por preocuparse de su patrimonio histórico y arqueológico con tanto esmero.

Con respecto a la pieza, el paisano que hizo entrega de ella al Ayuntamiento de Teba fue D. José Camarena. 
algo más de la mitad, aproximadamente ${ }^{15}$. Su leyenda aparece en relieve y está escrita en cúfico.

Solamente el anverso presenta restos de epigrafía (Fig. 3), mientras que el reverso, a priori, es anepigráfo y sin desbastar (Fig. 4) ${ }^{16}$. En el haz se puede leer el término mușālaha, o مصدالحة opacto de paz".

En el epígrafe hay dos letras que podemos distinguir: la primera de ellas es a (13 i), a la que le sigue ص (7 m), suficientes para proporcionarnos su lectura, [صد.]-لحة mușa[laha]. Este término se consigna habitualmente en los precintos con scriptio defectiva; es decir, sin el alif de prolongación.

Las medidas conservadas son las siguientes:

- Peso: 11’04 gr

Dimensiones conservadas: 24mm x 19 mm

- Grosor: 4'5 mm

\section{OTROS PRECINTOS DE SULH}

De los precintos de șulh, el más común es el que presenta el topónimo de Sevilla (SÉNAC, IBRAHIM, 2017: 32) puesto que seguramente era una de las ciudades más pobladas del reino visigodo. Musā b. Nușayr tomó la ciudad y pactó con sus representantes, aunque presentó alguna resistencia pasados estos primeros momentos.

Con respecto a los precintos sevillanos, sabemos que existen al menos una decena de ellos que han sido publicados por T. Ibrahim (2016) en los que se consignan los términos mușālahat Išbìliyya. Alguno de ellos se conserva en un museo local, como el de la colección museográfica de Gilena ${ }^{17}$ (Fig. 5).

De la actual Medina Sidonia, Šidūna, hay varios precintos, y al menos tres que se refieran a la capitulación pactada. Pero con respecto a
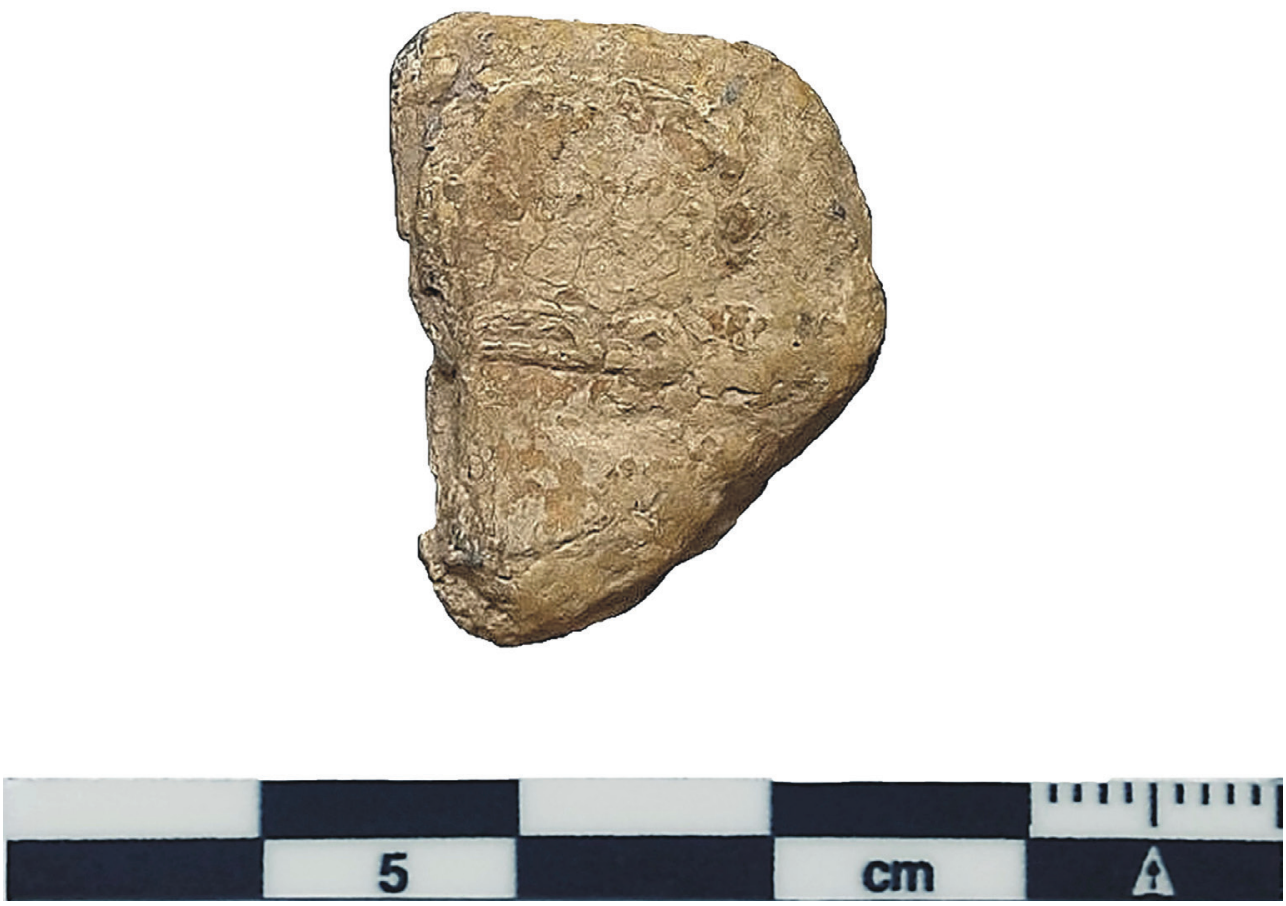

Fig. 3. Anverso del precinto con el término mușālaḥa. Foto: Pilar Delgado Blasco.

15. Para hacer tal afirmación hemos comparado este precinto con otros similares con respecto a las medidas (SÉNAC, IBRAHIM, 2017: 81-87).

16. Tal vez estuviese escrito, la leyenda se ha perdido y hoy es ilegible aunque, como decimos en el texto, aparece sin desbastar lo que indica que esta cara del precinto no estaba preparada para ser trabajada, como otro ejemplar publicado por T. Ibrahim (2011: 159, Fig. 17). O cabe la posibilidad de que la pieza haya sido seccionada, pero quizás esta cuestión sea descartable.

17. Uno muy parecido lo describe formalmente T. Ibrahim (1987: 707). 

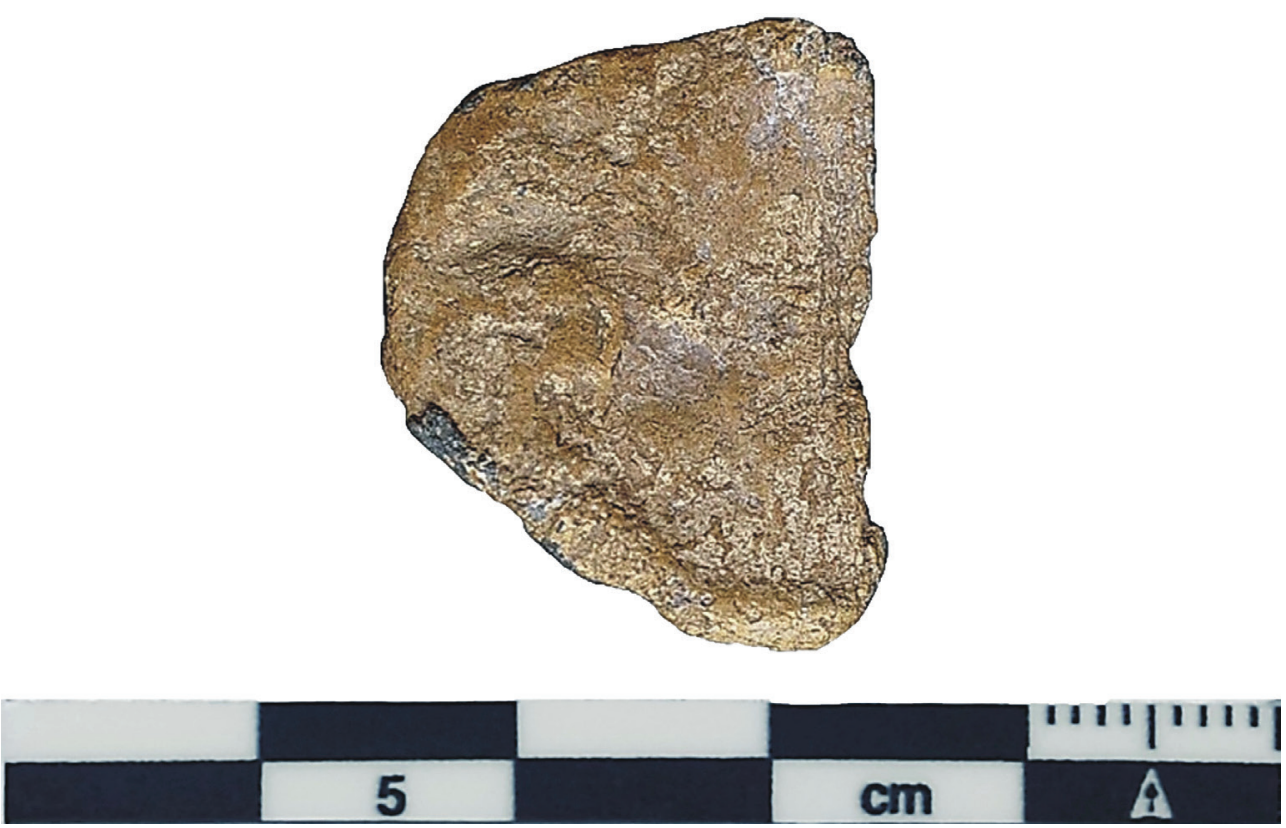

Fig. 4. Reverso del precinto: anepigráfico. Foto: José Carlos Escalante Gil.
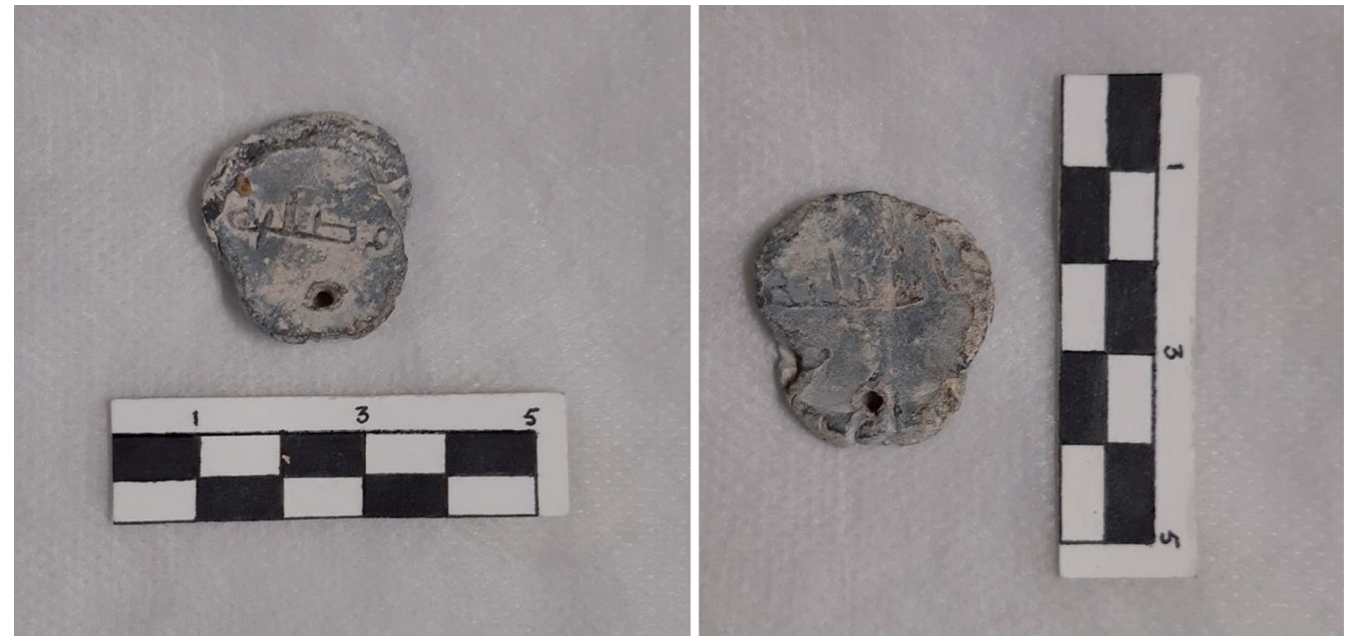

Fig. 5. Precinto de la Colección Museográfica de Gilena (Sevilla). Fuente: Colección Museográfica de Gilena (Sevilla).

este territorio, hay un sello que ha sido fechado en época califal y en el que llama la atención el vocablo kurā aunque, según se deriva de la lectura de la publicación, carece de contexto arqueológico y, por lo tanto, la datación resulta complicada solo a partir de la lectura del sello (MARTÍNEZ ENAMORADO, 2003b: 111).

Mușālahat ard Ŷyayān, es otra de las leyendas de estas piezas plúmbeas que hace alusión a la "tierra de Jaén", de la que se han publicado
3 ejemplares y en todas ellas aparece la misma leyenda con el término ard (AGÜERA CHAClNERO, 2020: 30-31).

Sobre el territorio de Elvira hay varios sellos, en algunos de ellos aparece la misma leyenda del que aquí nos ocupa, mușālahat Libira, y otro con la grafía jātim Ilbirra o precinto de Elvira, que seguramente estaría relacionado con el pago de la ŷizya (IBRAHIM, GASPARIÑO, 2016: 30). 
Como hemos visto, en muchos de los precintos de sulh/musālaha suelen aparecer los topónimos del lugar donde se sella el pacto, aunque también se sabe que el precinto de Nina Alta no es el único cuyo reverso se presenta sin epigrafiar (AGÜERA CHACINERO, 2020: 32, nº 9). En cuanto a los precintos "de paz" ocurre igual, solo el que presenta F. Agüera es parecido al de Nina Alta, si bien las referencias a lugares existen también en algunos sellos "de conquista por las armas", como el de Rayyo, con la leyenda fay' Allāh o la parte de Dios (SÉNAC, IBRAHIM, 2017a: 113), tratándose del primer precinto aparecido en la península ibérica de esta modalidad que hace referencia a un lugar concreto (GASPARIÑO, IBRAHIM, 2015: 7).

Otro plomo que no presenta grafía en su reverso es el que hace mención al ŷund de Hums (IBRAHIM, 2016: 33), asentado en Sevilla y Niebla (MANZANO MORENO, 1993: 330), en cuyo haz leemos day'at Hums ${ }^{18}$.

Hay dos sellos en cuyos reversos aparecen marcas, como el que está a nombre del emir, 'Abd al- Rahman b. Mu'āwiyya, en cuyo envés podemos observar la señal de la cota de malla (SÉNAC, IBRAHIM, 2017a: 77; LABARTA GÓMEZ, 2016: 275) o el que consigna la leyenda fay Allāh, en cuyo reverso se ve la marca de haber estado pegado a una tela.

Y, por último, otros dos sin leyendas en su cruz; uno con el término qad qusima (se ha repartido) (SÉNAC, 2017a: 101) y el sello en el que se puede leer al-wafā' li-lläh, "la fidelidad se le debe a Dios” (SÉNAC, IBRAHIM, 2017a: 125).

\section{EL YACIMIENTO DE NINA ALTA. MARCO GEOGRÁFICO Y ARQUEOLÓGICO (Fig. 6).}

Como hemos comentado, el sello apareció en el yacimiento arqueológico de Nina Alta que se encuentra al oeste de Teba. Esta localidad está ubicada en el extremo NO de la provincia de Málaga, formando parte del Surco
Intrabético, que es una de las principales rutas naturales de comunicación en sentido E-O de la zona montañosa de Andalucía.

Está regada por el río Guadalteba, que actúa de eje de unión entre la Depresión natural de Ronda y los Llanos de Antequera y que va siguiendo la propia disposición del Surco Intrabético.

Precisamente, tiene un buen acceso hasta Ronda por dos portillos bastante fáciles de flanquear, uno de ellos, y el más utilizado, se realiza a través de Cuevas del Becerro. Pero además, constituye un punto de comunicación importante entre la Bahía de Málaga y la Depresión del Guadalquivir, constituida por el río Guadalhorce, y la zona comprendida entre Ardales y Teba (GARCÍA, MARTÍNEZ, MORGADO, 1996: 24).

En cuanto a la Historia de este lugar, en lo que hoy es el término municipal de Teba, hay presencia humana desde el Paleolítico Medio hasta nuestros días, destacando yacimientos tan importantes desde el punto de vista arqueológico como la Cueva de las Palomas, el poblado de los Castillejos, las ruinas romanas del Cortijo del Tendedero, Cortijo del Tajo, el Castillo de la Estrella o Nina Alta que, concretamente, está ocupado desde la Prehistoria.

\section{NINA ALTA Y LA CORA DE TĀKURUNNĀ}

Nina Alta se ubica en lo que fue el distrito de Tākurunnā, del que son escasísimos los datos que aparecen en las fuentes escritas; unas fuentes que en ningún caso son anteriores al siglo X. Además, las citas sobre esta circunscripción hacen referencia siempre a algún hecho bélico. Las primeras noticias sobre ella pertenecen al momento en el que 'Abd al Rahman I al-Dājil marcha desde Elvira hasta Sevilla para reunir a seiscientos jinetes omeyas, nobles y mawlàs. Desde Torrox partió hacia Rayya y más tarde hacia Tākurunnā donde 'Abd al-A'là b. 'Awsaŷa, señor de esta cora, y los ŷundies que iban con él (con el emir) le prestaron juramento (DIKR, 


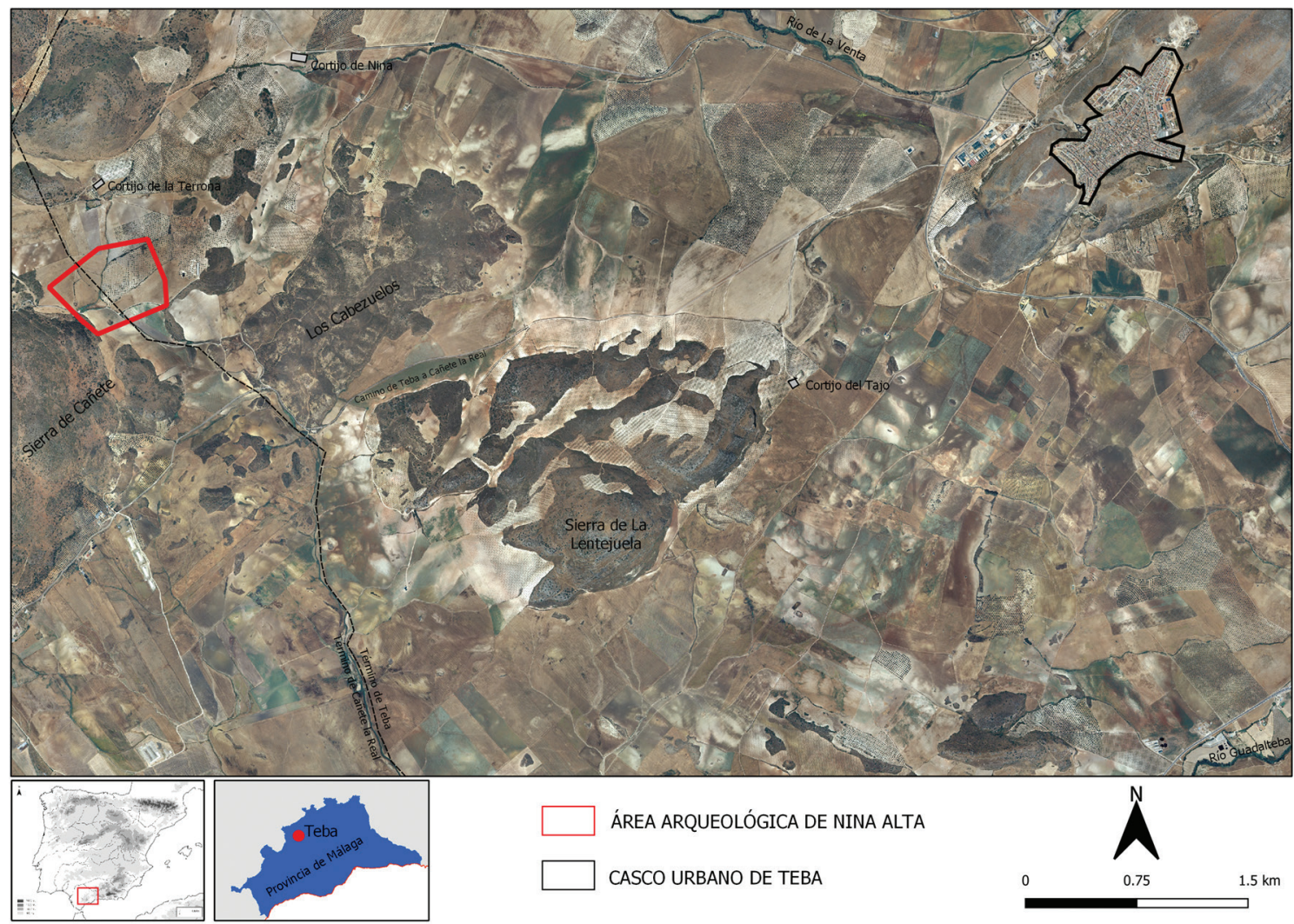

Fig. 6. Ubicación del yacimiento de Nina Alta (Teba, Málaga). Cortesía del Dr. Serafín Becerra Martín.

1983: 120 y FATH, 2002: 73); o el dato sobre la campaña contra Ŷilliqiya en 865, para la que la cora de Tākurunnā envía a 299 hombres frente a los 6500 hombres que envía la vecina cora de Rayya (SOUTO LASALA, 1995: 235). El distrito de Tākurunnā se circunscribiría a lo que en la actualidad es parte de la Serranía de Ronda junto con algunos territorios de la comarca del Guadalteba y parte de la Sierra de Cádiz, pero, ya hemos aclarado que, con respecto a esta cora, apenas contamos con información suficiente ni en las fuentes escritas y, menos aún, en las arqueológicas. Sobre estas, las únicas herramientas de análisis han sido dos prospecciones arqueológicas y el examen de los registros superficiales proporcionados por estos trabajos de campo CASTAÑO AGUILAR, 2006. No obstante, estas actuaciones han aportado datos importantes que han ayudado a determinar con relativa claridad, por ejemplo, los cambios que las poblaciones asentadas en estos lugares sufrieron tras la llegada de los invasores de allende el Estrecho (CASTAÑO AGUILAR, 2019: 440). Hecho que no disminuye la conveniencia de intervenir puntualmente en ciertos espacios que presentan indicios de poblamiento altomedieval para intentar aclarar algunas cuestiones sobre el tema.

Entre las coras (kuwar/ sing. kūra) de Rayya y Takurunnā hubo de encontrarse Teba, y entre hișn Ațība (Teba) y hiș̣n Qannīt (Cañete la Real) Nina Alta, yacimiento del que apenas tenemos datos de época medieval, aunque no es desconocido, ya que en él han aparecido importantes piezas arqueológicas presuntamente procedentes de este paraje (PÉREZ, MARTÍNEZ, 2019: 475) ${ }^{19}$.

19. En este artículo no vemos referencias explícitas al propio yacimiento de Nina Alta. Apenas si tenemos conocimiento sobre él ya que no se ha realizado una investigación desde el punto de vista científico que debería pasar, sin lugar a dudas, por una intervención arqueológica. Cualquier otra opinión estaría basada en las piezas aparecidas que, como bien se dice en el propio documento, son fruto de hallazgos casuales dentro de la delimitación del yacimiento arqueológico y que, por lo tanto, aportan una información sesgada sobre el yacimiento en sí. 
El lugar de Nina se ubica en una meseta formando un poljé y rodeado de montes calizos de escasa altura, en cuyo entorno se encuentra un importante acuífero. El espacio poblado se organiza alrededor de un qanāt central, de algo más de un kilómetro, labrado en piedra, que lo atraviesa. Su poblamiento fue bastante extenso en el tiempo, de lo que es buena muestra el hallazgo de monedas de distintas épocas: desde fulūs de la conquista hasta alguna pieza de época nazarí, aparte de monedas emirales y califales, como un lote de época de Almanzor, o monedas taifas de la Ceuta ham$m \bar{u} d \bar{i}^{20}$. Parece que hay numerosos vestigios en superficie, principalmente cerámicos, y bastantes silos excavados en la roca (MORGADO et alii 2001: 56 y 57). En este lugar no se han observado elementos fortificados de época andalusí, pero, como dijimos anteriormente, la aparición de restos arqueológicos proporciona el dato de una ocupación entre los siglos VIII y XV (MARTÍNEZ ENAMORADO, 2003a: 587).

\section{PARA CONCLUIR}

Hemos intentado poner en relación el precinto de plomo aparecido en el yacimiento de Nina Alta con la conquista de Hispania, concretamente en el territorio de lo que luego fue Takurunnā, de la que apenas tenemos referencias en las fuentes escritas, y de la que empezamos a contar con algunas procedentes de fuentes materiales.

En general, el tema de la conquista omeya ha significado un elemento de pugna historiográfica durante mucho tiempo precisamente por la escasez de información material. Esta materialidad, que hasta el momento la proporcionaban los precintos de plomo y las monedas acuñadas por los conquistadores, poco a poco se va completando con otros registros materiales, como el registrado arqueológicamente en la maqbara de Pamplona (FARO, GARCÍA BARBERENA y UNZU, 2007) ${ }^{21}$.

Volviendo al tema que nos ocupa, aunque carecemos de la suficiente información sobre Takurunnā, especialmente en el registro arqueológico, y está limitada a algunas hipótesis, en el presente texto se ha pretendido convertir estas hipótesis en consideraciones a partir de la lectura de un precinto de la conquista con la leyenda muṣālaha (مصدالحة), y del dato sobre su lugar de procedencia.

Precisamente, en relación con dichas consideraciones, es interesante resaltar el dato referido a los términos utilizados en los precintos. En este sentido, entre los sellos referentes a poblaciones del sur peninsular destacan las leyendas șulh/muṣālaha,como el de Nina Alta.

Los pactos de paz nos indican dos cosas: la primera, que en ese lugar hay capacidad de tributación y de control de población, puesto que se sobreentiende que se hacen con quien se puede, es decir, con alguien que tenga la posibilidad de poder afrontar los compromisos adquiridos en el tratado. Y la segunda, que estos moradores bajo el manto del aristócrata o señor visigodo pueden mantenerse en este territorio, al menos durante algún tiempo. Incluso la sustitución de un terrateniente visigodo por un señor árabe no parece que causase desalojos masivos de la población indígena (CHALMETA GENDRÓN, 2015: 64).

Así, se podría plantear la conclusión de que este territorio capituló ante las fuerzas árabes mediante pacto, con todo lo que ello implicaría para la comunidad allí asentada.

Pero también, y con todas las reservas, el precinto de Nina Alta, único por el momento aparecido en el territorio de la cora de Takurunnā, puede servir de argumento para proponer la moderada berberización de esta

20. Todas estas monedas referidas se encuentran expuestas en el Museo Municipal de Teba y proceden de hallazgos casuales y, hasta la fecha, no han sido estudiadas.

21. Donde se han hallado 190 tumbas sin superposiciones y depositadas en decúbito lateral derecho, mirando al E, en fosa simple y sin elementos de ajuar (DE MIGUEL, 2016: 130). La presencia musulmana en esta ciudad norteña se ha confirmado con una datación AMS que arrojó una fecha comprendida entre el 660 al 770 d. C. 
comarca, ya que, como han propuesto autores como J.M. CASTAÑO AGUILAR (2019: 446, nota 36), durante el período de conquista se llegó a acuerdos con los autóctonos del lugar, probando así que el poblamiento indígena continuará durante un período de tiempo.

A este mismo hecho alude M. Acién Almansa cuando refiere que la aristocracia visigoda, aunque con cierta debilidad social y económica, continúa ávida de poder y es normal que recurra a la práctica del pacto (ACIÉN ALMANSA, 1999: 56). Así, este argumento se nos presenta como una de las mejores y más coherentes propuestas con respecto a la "larga" pervivencia del poblamiento indígena hasta el contexto de la primera fitna y la consabida revuelta del caudillo 'Umar b. Hafsun.

Siguiendo este mismo hilo discursivo, para poder defender la pugna entre formaciones sociales tan dispares como la feudal y la islámica debemos aceptar que existe una persistencia de las comunidades indígenas tuteladas por la aristocracia, cuya continuidad seguirá basada en la posesión de tierras y en la renta (CASTAÑO AGUILAR, 2019: 424).

Este hecho entraría en contradicción con alguna propuesta en la que se afirma que la primera capital de esta comarca se habría asentado en lo que hoy es Nina Alta, en Teba, llamándose Madinnat Tākurunnā, un territorio en el que sus moradores serían mayoritariamente de origen beréber y que con los ifraníes cambió de ubicación y se estableció en Ronda (MARTÍNEZ ENAMORADO 2019: 135).

En vista de estas afirmaciones, podemos llegar a plantear que el sometimiento de este territorio se hizo por varios motivos, además del mero hecho de la conquista. El primero podría ser su ubicación: no olvidemos que geográficamente el yacimiento de Nina está estratégicamente situado puesto que conecta con la depresión del Guadalquivir, además de relativamente cercano a la costa malagueña y a la comarca de Antequera, y no hay que omitir su cercanía a Ronda.
Otro de dichos motivos pudo ser el económico ya que se trata de una zona productiva con una rica campiña, lo que implicaría un control de la producción, que estaría en manos de un aristócrata o señor, quien bien pudo ser el que capituló y llegó al acuerdo de paz, puesto que la necesidad de la recaudación propició este tipo de tratados.

Y por último, el político, porque es posible que el señor del lugar, al que se le rendía tributo, podría haber sido un personaje relevante, ya que se sobreentiende que los pactos se hacen con quien tiene capacidad para pactar, es decir, con alguien que tenga la posibilidad de poder afrontar los compromisos adquiridos en el tratado, que son esencialmente de carácter fiscal.

Así, la localización del precinto en el yacimiento de Nina Alta nos puede llevar a considerar que los procesos de islamización y de berberización en lo que, a la postre, fue Takurunnā, no debieron ser demasiado reseñables en un primer momento tras su ocupación.

Es muy probable que estas tierras de Tākurunnā siguieran en manos de sus poseedores locales durante algún tiempo tras la conquista (muy probablemente hasta la fitna), aun cuando sabemos del juramento que hace Ibn 'Awsaŷa ante 'Abd al-Raḥmān I. Sin embargo, no podemos afirmar que esta población fuese asimilada prontamente puesto que, aunque el potentado cambie, la población sigue asentada en su territorio. Esto nos llevaría a poner en cuarentena el tema del rápido anabolismo con el componente norteafricano en esta comarca.

Por último, otro asunto sería hasta qué punto se islamizaron los territorios de la antigua Hispania que no pactaron, y cómo fue ese proceso tras la entrada de los omeyas en la península ibérica, pues sabemos el momento en el que este proceso se ultima, ya en época califal. Después de muchos años parece que la arqueología y la epigrafía, como integrante de esta, poco a poco van respondiendo a alguna de estas cuestiones ${ }^{22}$.

22. Mi agradecimiento a la profesora Mª Antonia Martínez Núñez por si disposición y su asesoramiento continuo. 


\section{BIBLIOGRAFÍA}

DIKR, (1983): Dikr bilad al-Andalus o Una descripción anónima de al-Andalus, editada y traducida, con introducción, notas e índices, por Luis MOLINA, Madrid.

FATH, (2002): Fath al-Andalus, traducción de Mayte Penelas, Madrid.

ACIÉN ALMANSA, Manuel (1999): "Poblamiento indígena en al-Andalus e indicios del primer poblamiento andalusí". al-Qantara, 20, n 1, pp. 47-64. https://doi.org/10.3989/alqantara.1999.v20. i1.451

AGÜERA CACHINERO, Felipe (2020): "Recopilación de precintos árabes de plomo. Adición al Corpus de Precintos Andalusíes". Manquso, 11, pp. 25-44.

ARIZA ARMADA, Almudena (2016): "Los dinares bilingües de al-Andalus". Hécate, 3, pp. 137-158.

ARIZA ARMADA, Almudena (2017): "Del sólido al dinar. En torno a las primeras emisiones áureas del Magreb (76/695-696 - 100/718719). Nuevas perspectivas". Hécate, 4, pp. 88-113.

BATES Michael L. (1992): "The coinage of Spain Under the Umayyad Caliphs of the East., III Jarique de Numismática Hispano-Árabe, pp. 271-289. Madrid.

CANTO GARCÍA, Alberto (2011): "Las monedas de la conquista". Zona Arqueológica, 15, 711, Arqueología entre dos mundos, I, pp. 132-143.

CANTO, Alberto e IBRAHIM, Tawfiq (2004): Moneda andalusí. La colección del Museo de la Casa de la Moneda. Madrid.

CASTAÑO AGUILAR, José Manuel (2004): "Poblamiento medieval en la Serranía de Ronda. Campaña de prospección arqueológica superficial en el Valle del Genal". Anuario Arqueológico de Andalucía, 2001, pp. 49-59. Sin publicar.

CASTAÑO AGUILAR, José Manuel (2006): "Prospección arqueológica superficial en la depresión natural de Ronda. Informe Preliminar de la Campaña de 2003-2005". Informe presentado a la Dirección General de Bienes Culturales de la Consejería de Cultura. Junta de Andalucía.

CASTAÑO AGUILAR, José Manuel (2019): La Serranía de Ronda. Entre la Antigüedad y la Edad Media. Jaén: UJA Editorial.

CHALMETA GENDRÓN, Pedro (1994): Invasión e islamización. La conquista de Hispania y la formación de al-Andalus. Madrid.

CHALMETA GENDRÓN, Pedro (2013): "Derecho y práctica fiscal musulmana: el primer siglo y medio". En BALLESTÍN, X. y PASTOR, E. (eds.), Lo que vino de Oriente. Horizontes, praxis y dimensión material de los sistemas de dominación fiscal en Al-Andalus (ss. VII-IX). Oxford, BAR International Series 2525, pp. 1-16.

CHALMETA GENDRÓN, Pedro (2015): "Los primeros 46 años de economía andalusí". Alhadra, 1, pp. 41-88.

COLLINS, Roger (1991): La conquista árabe (710-797). Barcelona.

DE MIGUEL-IBÁÑEZ, María de la Paz (2016): La maqbara de Pamplona (s. VIII). Aportes de la osteoarqueología al conocimiento de la islamización en la marca superior. Tesis doctoral. Universidad de Alicante.
DOMENÉ SÁNCHEZ, Domingo (2011): Año 711. La invasión musulmana de Hispania. Madrid.

FARO, José Antonio, GARCÍA-BARBERENA, María y UNZU, Mercedes (2007): "La presencia musulmana en Pamplona”. En Sénac, Ph. (coord.): Villes et campagnes de Tarraconaise et d'al-Andalus (VIe-Xle siècles): la transition, pp. 97-138. París. https://doi.org/10.4000/ books.pumi. 25663

FIERRO BELLO, M.I (2018): "El caso del cristiano que quería ser ejecutado". https://www.alandalusylahistoria.com/?p=274 consultado el 27 de mayo de 2020, 17:33.

GARCÍA, Eduardo, MARTÍNEZ, Virgilio y MORGADO, Antonio (1996): El Bajo Guadalteba (Málaga): Espacio y Poblamiento. Una aproximación a Teba y su entorno. Málaga.

GARCÍA SANJUÁN, Alejandro (2004): "Las causas de la conquista islámica de la península Ibérica según las crónicas medievales". Miscelánea de Estudios Árabes y Hebraicos. Sección Árabe-Islam, 53, pp. 101-127.

GARCÍA SANJUÁN, Alejandro (2013): La conquista islámica de la península ibérica y la tergiversación del pasado. Madrid.

GASPARIÑO, Sebastián e IBRAHIM, Tawfiq (2015): "Adiciones a los precintos de la Conquista: ¿Rayyo?”. Manquso, 1, pp. 7-10.

GASPARIÑO, Sebastián e IBRAHIM, Tawfiq (2019): "Nuevo precinto del "pacto de paz" mușālaha de lugar desconocido". Manquso, 10, pp. 5-6.

GUICHARD, P. (2002): De la expansión árabe a la Reconquista. Esplendory fragilidad de al-Andalus. Granada.

GUTIÉRREZ LLORET, Sonia (2011): "El reconocimiento arqueológico de la islamización. Una mirada desde al-Andalus". Zona Arqueológica, 15, 711 Arqueología entre dos mundos, I, pp. 191-210.

GUTIÉRREZ LLORET, Sonia (2014): "La materialidad del Pacto de Teodomiro a la luz de la arqueología". eHumanista/IVITRA, 5, pp. 262-288.

IBRAHIM, Tawfiq (1987): "Evidencias de precintos y amuletos en al-Andalus". II Congreso de Arqueología Medieval Española, pp. 705-710.

IBRAHIM, T. (1995): "Un precinto a nombre de 'Abd al-Rahmān I". al-Qanțara, XVI/1, pp. 143-146.

IBRAHIM, Tawfiq (2011): "Nuevos documentos sobre la Conquista Omeya de Hispania: Los precintos de plomo». Zona Arqueológica, 15, 711 Arqueología e Historia entre dos mundos, I, pp. 146-161.

IBRAHIM, Tawfiq (2016): "Los precintos de la conquista y el dominio Omeya de Hispania". Manquso, 4, pp. 7-38.

IBRAHIM, Tawfiq y GASPARIÑO, Sebastián (2016): "Adiciones a los precintos de la Conquista: Córdoba, Elvira y una variante de al-Andalus". Manquso, 4, pp. 39-42.

KENNEDY, Hugh (2007): Las grandes conquistas árabes. Barcelona.

LABARTA GÓMEZ, Ana (2016): "Parada militar en la Córdoba omeya y restos arqueológicos". Mainake, XXXVI, pp. 263-278.

LORENZO JIMÉNEZ, Jesús (2011): "Tras las huellas de los conquistadores”. Andalucía en la Historia, 30, pp. 28-31. 
MANZANO MORENO, Eduardo (1993): "Los ŷund sirios en al-Andalus". Al- Qantara, XIV, pp. 327-359.

MANZANO MORENO, Eduardo (2000): "La conquista del 711: transformaciones y pervivencias". En L. Caballero y P. Mateo (eds.), Visigodos y Omeyas. Un debate entre la Antigüedad Tardía y la Alta Edad Media, pp. 401-404. Madrid.

MARTÍNEZ ENAMORADO, Virgilio (2003a): Al-Ándalus desde la periferia. La formación de una sociedad musulmana en tierras malagueñas. Málaga.

MARTÍNEZ ENAMORADO, Virgilio (2003b): "Un plomo con la leyenda en árabe Kürat sadūna". Almajar, 1, pp. 111-113.

MARTÍNEZ ENAMORADO, Virgilio (2004); "Una primera propuesta de interpretación para los plomos con epigrafía árabe a partir de los hallazgos de Nina Alta (Teba, provincia de Málaga)". Al-Andalus-Magreb, 10, pp. 97-127.

MARTÍNEZ ENAMORADO, Virgilio (2019): "Una propuesta de tabla sobre la geografía tribal de la Serranía de Ronda". Actas II CIHSR, Anejos de Takurunnā, n² 2, pp. 131-148.

MARTÍNEZ NÚÑEZ, Ma Antonia (1997): "Escritura árabe ornamental y epigrafía andalusí". Arqueología y Territorio Medieval, nº 4, pp. 127-162. https://doi.org/10.17561/aytm Muchas gracias

MARTÍNEZ NÚÑEZ, Ma Antonia (2001): "Epigrafía monumental y élites sociales en al-Andalus". Nakla, 17. Epigrafía árabe y arqueología medieval, pp. 19-60.

MARTíNEZ NÚÑEZ, Ma Antonia (2011): "Por qué llegaron los árabes a la península ibérica: Las causas de la conquista musulmana de 711". Awraq, 3, pp. 21-36.

MORGADO Antonio, MARTíNEZ Virgilio y GARCÍA Eduardo (2001): "El Museo Arqueológico Municipal de Teba". En Revista de Arqueología, 240, pp. 50-57.
ORTEGA ORTEGA, Julián M. (2018): La conquista islámica de la península Ibérica. Una perspectiva arqueológica. Madrid.

PÉREZ, José Manuel y MARTínEZ Virgilio (2019): "Evidencias arqueológicas de magia talismánica en Nina Alta (Teba, Málaga). En Actas del II congreso internacional historia de la Serranía de Ronda. Entre al- Andalus y los inicios de la Edad Moderna, pp. 473-496.

RÉBE Isabelle, RAYNAUD Claude y SÉNAC Philippe (2015): Le premier Moyen Age à Ruscino (Château- Roussillon, Perpignan, Pyrénées-Orientales) entre Septimanie et al-Andalus (VI/e-Xlé). Mompellier.

SÉNAC, Philippe (2009): "Nota sobre la conquista musulmana de la Narbonense (siglo VIII)". XI Congreso de Estudios Medievales. Cristianos y musulmanes en la península Ibérica: la guerra, la frontera y la convivencia, pp. 165-176.

SÉNAC, Philippe (2012): "Aux confins d'al-Andalus (VIIle siècle): histoire et archéologie de la conquête de la Tarraconaise orientale et de la Narbonnaise ». 711, Arqueología e Historia entre dos mundos, pp. 177-185.

SÉNAC Philippe, GASC Sébastien, MELMOUX Pierre-Yves y SAVARESE Laurent (2014): "Nouveaux vestiges de la présence musulmane en Narbonnaise au VIIle siècle". al-Qantara, XXXV/ 1, pp. 61-94. https://doi.org/10.3989/alqantara.2014.003

SÉNAC, Philippe e IBRAHIM Tawfiq (2017a): Los precintos de la conquista omeya y la formación de al-Andalus (711-756). Granada.

SÉNAC, Philippe e IBRAHIM Tawfiq (2017b): "Notes sur des sceaux de la conquête omeyyade (première moitié VIIle siècle)". Mélanges en l'honneur du Professeur Jean-Claude Cheynet, pp. 439-450.

SOUTO LASALA, Juan Antonio (1995): "El emirato de Muhammad I en el "Bayān al- Mugrib" de Ibn 'Idāri”. Anaquel de estudios árabes, 6, pp. 209-248. 